\title{
Multi-wavelength characterization of the outskirts of spiral galaxies
}

\author{
Judit Bakos \& Ignacio Trujillo \\ Instituto de Astrofísica de Canarias, \\ C/ Vía Lactea, La Laguna, 38205, Tenerife, Spain \\ email: jbakos@iac.es, trujillo@iac.es
}

\begin{abstract}
We have explored late-type spiral galaxies with truncated radial surface brightness profiles. Based on the study of optical color profiles, Bakos et al (2008) suggested that truncated (TYPE II) galaxies have a generally older stellar population in their outer regions: one observable consequence of this would be a strong dependence of the structural parameters of the outer disk on the observing wavelength. To corroborate this result, we obtained surface brightness profiles using data from GALEX(UV), SDSS(optical), UKIDSS(NIR) and SPITZER-IRAC(IR). We have characterized the behaviour of the outer disk by obtaining the ratio of the inner and outer scale-lengths. Here we show the example of NGC0450. Our results suggest that there is an existing general trend of the scale-length ratio: from bluer to redder bands the scale-length ratio decreases, which is in accordance with the idea of the old smooth stellar disk in the outer disk.
\end{abstract}

Keywords. Keyword1, keyword2, keyword3, etc.

\section{Introduction}

The radial surface brightness profiles can exhibit three distinct forms. Type I profiles can be fitted with one exponential fitting function (de Vaucouleurs 1993). Type II profiles contain a down-bending exponential after the break (Freeman 1970; van der Kruit 1979). Profiles with up-bending outer exponential are the Type III (Erwin et al. 2005) profiles. However different these galaxies are regarding their radial surface brightness profiles, they do not differ morphologically. (See Online Material of Pohlen \& Trujillo 2006).

The presence of stars in the outer regions of a galaxy disk is not well explained by current star-formation theories (Kennicutt (1989, Elmegreen \& Parravano, Schaye 2004), which predict no stars should be forming (i.e. at regions where the gas density dropped below $10 M_{\odot} p c^{-2}$ ). These stars are very often located beyond the break radius of Type II galaxies. In order to investigate how the star formation progresses in the different parts of the disks, Bakos et al (2008) studied the color and stellar mass ditribution of a large sample of disk galaxies, where they found have that truncated galaxies have an older stelar population in the outskirts, meanwhile it is not accompanied with a significant drop in the stellar mass. An observable consequence of this is the wavelength dependence of the inner-outer scale-length ratio, which whould decrease towards redder wavelength as we approach to tackle the old stellar disk.

\section{Multi-wavelength characterization of a truncated disk}

We have chosen galaxies with full wavelength coverage (from UV to IRAC) from the PT06 sample. Here we show an example of NGC0450. We have obtainded the radial surface brightness profiles in all bands using fixed isophotes of the ellipticity and position angle. We first masked the stars and other background objects on the galaxy image, the objects were identified by sextractor. After masking we calculated the moments of the light distribution of the galaxy in r'-band, which is sufficiently smooth enough to derive 


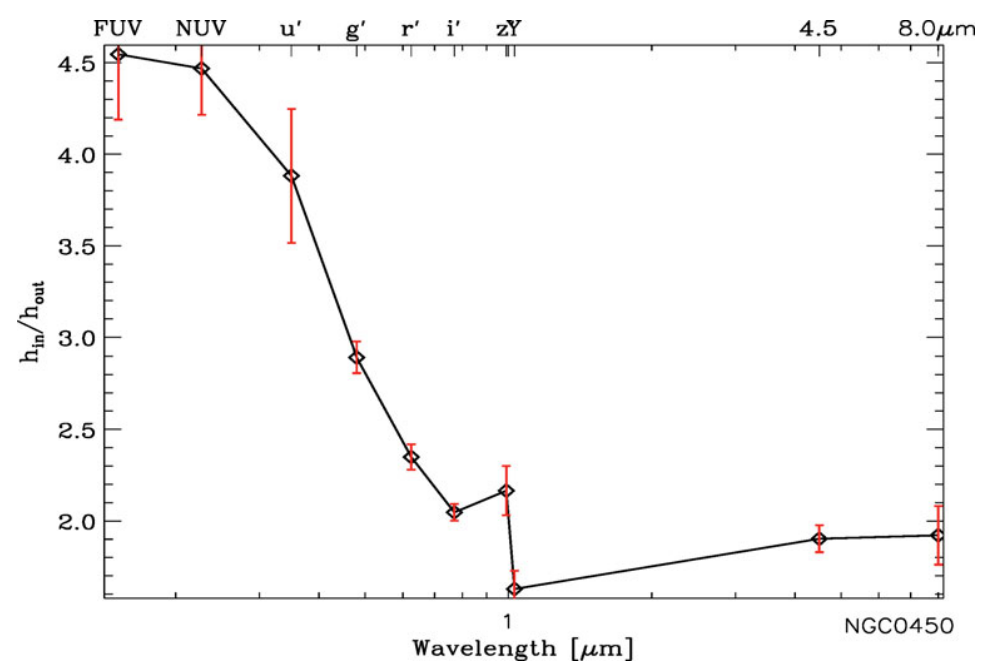

Figure 1. The dependence of the scale-length ratio on wavelength in case of NGC0450. The decrease on the ratio towards redder wavelength is quite dramatic. Errorbars are propagated errors coming from the errors on the fit. We fitted the surface brightness profiles by using robust linefitting method.

the correct position angle and ellipticity of the galaxy. For NGC0450 our position angle on the SDSS image (from the $\mathrm{X}$-axis towards $\mathrm{N}$ ) is -6.5 degrees, and the ellipticity is 0.25 . The break radius if this galaxy independently of wavelength is $\sim 70$ arcseconds. (See Pohlen \& Trujillo 2006.)

We characterize the outer disk by means of calculating the ratio of the inner and outer scale-lengths. We use profiles which are deep enough to obtain reliable fit beyond the break. We find that the scale-length ratio decreases towards redder wavelengths. (See Fig. 1.)

Discussion The inner-outer scale-length ratio of NGC0450, which is galaxy with truncated (TYPE II) surface brightness profile, depends on the observing wavelength. There is a dramatic decrease towards redder wavelength which supports the idea suggested in Bakos et al. (2008) that in the outer regions of truncated galaxies the change on the slope of the surface brightness is in reality caused by a change in the stellar population: having a very few young stars, the outer disk is dominated by the old stellar disk.

\section{References}

Bakos, J., Trujillo, I., \& Pohlen, M. 2008, ApJ, 683, L103

de Vaucouleurs, G. 1958, ApJ, 128, 465

Elmegreen, B. G. \& Parravano, A. 1994, ApJ, 435, L121

Erwin, P., Beckman, J. E., \& Pohlen, M. 2005, ApJ, 626, L81

Freeman, K. C. 1970, ApJ, 160, 811

Kennicutt, R. C. 1989, ApJ, 344, 685

Pohlen, M. \& Trujillo, I. 2006, A\&A, 454, 759 (PT06)

Schaye, J. 2004, ApJ, 609, 667

van der Kruit, P. C. $1979 A \& A S 38,15$ 